\title{
Mean Acquisition Time Analysis of Fixed-Step Serial Search Algorithms
}

\author{
Sinan Gezici, Member, IEEE
}

\begin{abstract}
In this paper, mean acquisition time (MAT) analysis of fixed-step serial search (FSSS) algorithms is presented. First, it is shown that the MAT of an FSSS algorithm can be obtained from that of a conventional serial search (CSS) algorithm after a certain mapping of the uncertainty region. Then, a generic formula for the MAT of FSSS algorithms is derived, which is valid for both dense and sparse channel environments. In addition, MAT formulas for high signal-to-noise ratio scenarios, for large uncertainty regions, and for dense channels are obtained as special cases of the generic solution. Finally, simulation results are presented to verify the analysis and to investigate the factors that affect the optimal step size for FSSS algorithms.
\end{abstract}

Index Terms-Acquisition, mean acquisition time (MAT), conventional serial search (CSS), fixed-step serial search (FSSS).

\section{INTRODUCTION}

A SPREAD spectrum receiver first needs to perform sequence synchronization before data demodulation can commence. This sequence synchronization is performed by a coarse sequence alignment stage, called acquisition, followed by a fine alignment stage, called tracking [1]. The main task of an acquisition algorithm is to coarsely determine the correct sequence phase of an incoming signal with respect to a local reference (template) signal at the receiver. In order to determine the correct phase, the receiver needs to test a number of possible sequence phases, which are also called cells. The cells for which the template signal and the incoming signal have the same phase are called in-phase cells. Due to multipath propagation, there can be multiple in-phase cells in a system, in which case the acquisition process can stop whenever one of the in-phase cells is detected.

Acquisition algorithms can be broadly classified into serial and parallel schemes. In a parallel acquisition algorithm, multiple sequence phases are tested simultaneously, whereas a serial acquisition algorithm tests one cell at a time. The selection between parallel and serial schemes mainly depends on performance and cost trade-offs. The main focus of this work is on serial search acquisition algorithms, which offer low cost solutions to the acquisition problem.

A common criterion for performance of acquisition algorithms is the mean acquisition time (MAT), which defines the average time until acquisition is achieved [2]. In order to minimize the MAT, both the search algorithm, which determines the order in which the possible phases are searched, and the decision rule, which determines whether a given cell is an inphase cell, should be optimized. In order to minimize the MAT

Manuscript received January 31, 2008; revised August 8, 2008; accepted November 4, 2008. The associate editor coordinating the review of this paper and approving it for publication was L. Yang.

S. Gezici is with the Department of Electrical and Electronics Engineering, Bilkent University, Bilkent, Ankara TR-06800, Turkey (e-mail: gezici@ee.bilkent.edu.tr).

Digital Object Identifier 10.1109/TWC.2009.080147 for a given decision rule, various serial search algorithms can be considered. The conventional serial search (CSS) algorithm searches the possible cells consecutively until acquisition is achieved [2]. On the other hand, other techniques such as fixed-step serial search (FSSS) and bit reversal algorithms test the cells in specific non-consecutive orders [3], [4], [5].

In the presence of a single in-phase cell in the uncertainty region, which is defined as the index set corresponding to all possible phases of the incoming signal, the order of the search is not important, and the serial search algorithms have the same MAT. The MAT formula for the case of a single in-phase cell is derived in [2]. In the presence of multiple in-phase cells, the search algorithm becomes an important factor in determining the MAT. In [3], it is shown that the FSSS and the bit-reversal algorithms outperform the CSS algorithm in the absence of noise in the system. The MAT of the CSS algorithm for multiple in-phase cells is derived in [6] by assuming that the search always starts at an outof-phase cell. For an uncertainty region with identical and consecutive in-phase cells, an optimization theory framework is established in [5] and [7] to obtain upper and lower bounds on MATs of serial search algorithms, and to show that the FSSS algorithm achieves a near-optimal MAT. In [8], MAT formulas for various serial search algorithms are provided, but the analysis implicitly assumes that the first in-phase cell position is fixed for all cases, which can result in different MATs from those for practical scenarios, as stated in [5]. Finally, [9] proposes energy combining from multiple cells in order to increase acquisition performance of ultra-wideband (UWB) systems.

The main purpose of this work is to provide a generic closed-form MAT expression for the FSSS algorithm. As studied in [5], the FSSS algorithm has a near-optimal MAT in certain scenarios; hence, it is important to have a closedform MAT expression of that scheme for system and algorithm design purposes. Unlike previous studies, the current work considers a generic framework, in which the in-phase cells do not have to be identically distributed or consecutive (i.e., the multipath channel can be dense or sparse with any power delay profile), the serial search can start from any cell including the in-phase cells, and the averaging over various values of the first in-phase cell position is performed in order to obtain realistic MATs. In order to provide such a generic MAT expression, it is first shown that averaging over the various positions of the first in-phase cell corresponds to a mapping of the FSSS MAT calculation problem into a CSS MAT calculation problem for a modified uncertainty region. Then, generic MAT formulas are obtained and simple approximations are presented for special cases (Section III). Simulation results are presented in order to verify the theoretical analysis and 
to investigate the optimal step size of the FSSS algorithm for various systems parameters (Section IV). Finally, concluding remarks are made (Section V), and implications of the study regarding the derivation of generic MAT expressions for any serial search algorithm are discussed.

\section{System Model and Serial Search Techniques}

Let $N$ and $L$ represent the total number of cells in the uncertainty region and the number of in-phase cells, respectively. The set of indices for the uncertainty region can be expressed as

$$
\mathcal{U}=\{1,2, \ldots, N\}
$$

Similarly, the indices for the in-phase cells are given by

$$
\tilde{\mathcal{L}}=\left\{\tilde{l}_{1}, \tilde{l}_{2}, \ldots, \tilde{l}_{L}\right\}
$$

where $\tilde{l}_{i} \in\{1,2, \ldots, N\}$ represents the index for the $i$ th inphase cell, for $i=1,2, \ldots, L$. Note that the in-phase cells do not have to be adjacent in this formulation; therefore, the analysis considers both sparse and dense channels.

For a given multipath channel profile, $\tilde{l}_{1}$ can take any value in $\mathcal{U}$ with equal probability, assuming that there is no prior information about the phase delay of the incoming signal.

For an uncertainty region as in (1), a serial search algorithm tests one cell at a given time. If the signal is detected in an in-phase cell, the acquisition state is reached and the search stops. Otherwise, the next cell is tested and that procedure continues until the acquisition state is reached. Whenever an out-of-phase cell is declared as an in-phase cell due to noise, which is called a false alarm (FA), additional verification tests are performed. If the cell is in fact an out-of-phase cell, it is assumed that the verification stage uncovers that. However, the verification process causes an additional time delay, which is called the penalty time for an FA. Then, the search continues with the next cell. It is assumed that each out-of-phase cell has the same FA probability for a given system [2], [6].

The testing order of a serial search is determined by the search strategy. For example, the CSS algorithm searches for an in-phase cell by starting from the first index and searching all the cells sequentially until acquisition. On the other hand, an FSSS algorithm with a step size of $M$, denoted by FSSS$M$, searches the cells in the following order:

$$
\mathcal{S}=\{1,1 \oplus M, 1 \oplus 2 M, \ldots, 1 \oplus(N-1) M\},
$$

where $x \oplus y$ represents a modulo- $N$ addition defined as $x \oplus y=$ $x+y-k N$ for some unique integer $k$ such that $x+y-k N \in$ $\mathcal{U}$. Note that $M$ and $N$ should be relatively prime to ensure that all the cells in the uncertainty region $\mathcal{U}$ can be tested by the FSSS- $M$ algorithm [5]. Also note that FSSS- $M$ algorithm reduces to the CSS algorithm for $M=1$.

\section{MAT ANALYSIS}

\section{A. Relation between CSS and FSSS Algorithms}

In order to obtain a closed-form expression for the MAT of FSSS algorithms, the following proposition will be used.

Proposition 1: Let $\tilde{\mathcal{L}}=\left\{\tilde{l}_{1}, \tilde{l}_{2}, \ldots, \tilde{l}_{L}\right\}$ denote the positions of the in-phase cells in an uncertainty region of $N$ cells. For

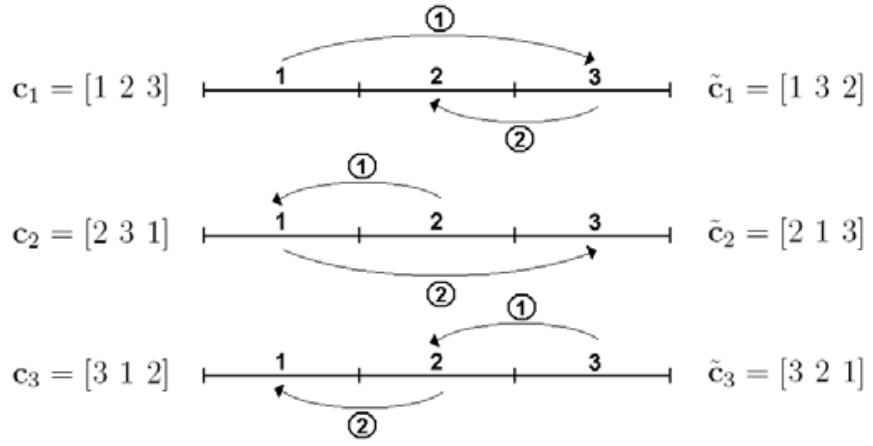

Fig. 1. The FSSS-2 algorithm for $N=3$. For three different starting cells, three different search orders are obtained. Overall, the algorithm corresponds to the CSS algorithm when the cells are re-organized since the new cell structures are circularly shifted versions of each other.

this scenario, the MAT of an FSSS-M algorithm is equal to that of a CSS algorithm for a scenario in which the in-phase cell positions are given by

$$
\mathcal{L}=\left\{l_{j} \in\{1,2, \ldots, N\} \mid \tilde{l}_{j}=1 \oplus\left(l_{j}-1\right) M, j=1, \ldots, L\right\} .
$$

Proof: An intuitive explanation of the proposition is provided in Fig. 1. In the following, a mathematical proof is developed.

The MAT of the FSSS- $M$ algorithm for a given set of inphase cell indices $\tilde{\mathcal{L}}$ is expressed as

$$
\text { MAT }=\frac{1}{N} \sum_{i=1}^{N} \mathrm{E}\left\{T \mid \mathbf{c}_{i}, \tilde{\mathcal{L}}_{\mathrm{r}}, \mathcal{S}\right\},
$$

where $\mathrm{E}\left\{T \mid \mathbf{c}_{i}, \tilde{\mathcal{L}}_{\mathrm{r}}, \mathcal{S}\right\}$ represents the MAT for the serial search algorithm represented by $\mathcal{S}$ in (3) when the cells, denoted by $\mathbf{c}=\left[\begin{array}{llll}c_{1} & c_{2} & \cdots & c_{N}\end{array}\right]$, are investigated starting from the $i$ th one, i.e., $\mathbf{c}_{i} \doteq\left[\begin{array}{lll}c_{i} \cdots c_{N} & c_{1} \cdots c_{i-1}\end{array}\right]$ (c.f. Fig. 1), and the relative positions of the in-phase cells are specified by $\tilde{\mathcal{L}}_{\mathrm{r}}=\left\{1, \tilde{l}_{2,1}, \ldots, \tilde{l}_{L, 1}\right\}$ with $\tilde{l}_{j, 1}=\tilde{l}_{j} \oplus\left(1-\tilde{l}_{1}\right)$ for $j=1,2, \ldots, L$. Since the index $i$ is uniformly distributed in $\{1, \ldots, N\}$, the overall MAT is calculated by the averaging operation in (5).

Since the FSSS- $M$ algorithm searches the cells in steps of $M$ cells according to $\mathcal{S}$, (5) becomes

$$
\text { MAT }=\frac{1}{N} \sum_{i=1}^{N} \mathrm{E}\left\{T \mid \tilde{\mathbf{c}}_{i}, \mathcal{L}_{\mathrm{r}}, \mathcal{U}\right\},
$$

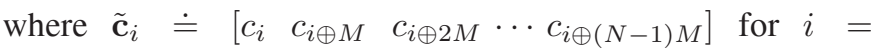
$1,2, \ldots, N$ (c.f. Fig. 1), $\mathcal{U}=\{1,2, \ldots, N\}$, and $\mathcal{L}_{\mathrm{r}}=$ $\left\{1, l_{2,1}, \ldots, l_{L, 1}\right\}$ with $l_{j, 1}=l_{j} \oplus\left(1-l_{1}\right)$ and $\tilde{l}_{j}=1 \oplus$ $\left(l_{j}-1\right) M$ for $j=1,2, \ldots, L$. In other words, searching the cells in $\mathbf{c}_{i}$ according to $\mathcal{S}$ for a given in-phase cell profile $\tilde{\mathcal{L}}_{\mathrm{r}}$ is the same as searching the cells in $\tilde{\mathbf{c}}_{i}$ sequentially, i.e., according to $\mathcal{U}$, for the in-phase cell profile $\mathcal{L}_{\mathrm{r}}$.

Finally, it can be shown that $\tilde{\mathbf{c}}_{1}, \tilde{\mathbf{c}}_{2}, \ldots, \tilde{\mathbf{c}}_{N}$ are all distinct 


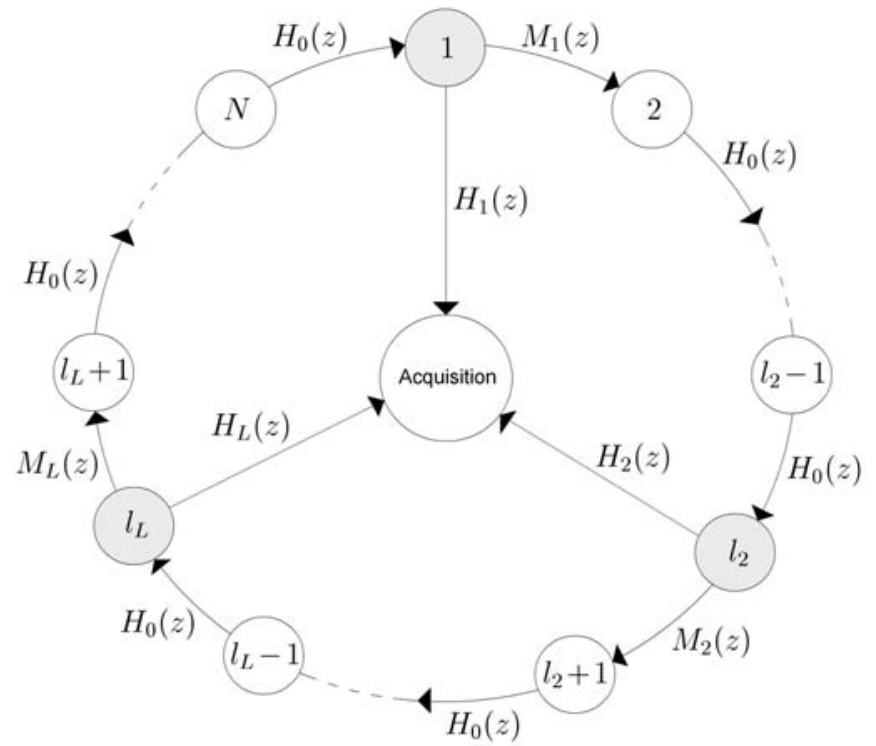

Fig. 2. Flow graph diagram for an uncertainty region of $N$ cells, where $\left\{1, l_{2}, \ldots, l_{L}\right\}$ represent the indices of the in-phase cells.

and circularly shifted versions of each other. ${ }^{1}$ Therefore, (6) corresponds to the MAT of a CSS algorithm for $\mathcal{L}_{\mathrm{r}}$ and the cell order specified by $\left[\begin{array}{lllll}c_{1} & c_{1 \oplus M} & c_{1 \oplus 2 M} & \cdots & c_{1 \oplus(N-1) M}\end{array}\right]$ without loss of generality. In other words, (6) can be expressed as

$$
\operatorname{MAT}=\frac{1}{N} \sum_{i=1}^{N} \mathrm{E}\left\{T \mid l_{1}=i, \mathcal{L}_{\mathrm{r}}, \mathcal{U}\right\},
$$

and $l_{j}$ is given by the relation $\tilde{l}_{j}=1 \oplus\left(l_{j}-1\right) M$ for $j=$ $1,2, \ldots, L$, which completes the proof.

Proposition 1 implies that the MAT of the FSSS- $M$ algorithm can be calculated in two steps. First, the positions of the in-phase cells are mapped into a new set according to (4). Then, the MAT of a CSS algorithm is calculated for the new in-phase cell positions, which is equal to the MAT of the FSSS- $M$ algorithm for the original in-phase cell positions. Therefore, obtaining a generic formula for the MAT of FSSS algorithms is equivalent to obtaining a formula for the MAT of a CSS algorithm for a given set $\mathcal{L}=\left\{l_{1}, l_{2}, \ldots, l_{L}\right\}$ of in-phase cell positions.

\section{B. Generic MAT Formulas}

In order to calculate the MAT of a CSS algorithm, the flow graph approach can be employed [2], [6]. Unlike previous studies, the formulation in this paper considers arbitrary positions for the in-phase cells specified by $\mathcal{L}=\left\{l_{1}, l_{2}, \ldots, l_{L}\right\}$ (Fig. 2), instead of assuming adjacent in-phase cells [5], [6]. In other words, the derivations in this section are valid not only for dense channels but also for sparse channels.

Let $H_{0}(z)$ represent the transfer function ("path gain") from an out-of-phase cell to the next cell, which is given

\footnotetext{
${ }^{1}$ Note that this is not true in general for other search algorithms than the FSSS. For example, for the search algorithm specified by $\tilde{\mathcal{S}}=\{1,3,4,2\}$, $\tilde{\mathbf{c}}_{1}, \tilde{\mathbf{c}}_{2}, \tilde{\mathbf{c}}_{3}$ and $\tilde{\mathbf{c}}_{4}$ are given, respectively, by $\left[\begin{array}{llll}c_{1} & c_{3} & c_{4} & c_{2}\end{array}\right],\left[\begin{array}{llll}c_{2} & c_{4} & c_{1} & c_{3}\end{array}\right]$, $\left[\begin{array}{llll}c_{3} & c_{1} & c_{2} & c_{4}\end{array}\right]$ and $\left[\begin{array}{llll}c_{4} & c_{2} & c_{3} & c_{1}\end{array}\right]$, which are not circularly shifted versions of each other.
}

by $H_{0}(z)=\left(1-P_{\mathrm{FA}}\right) z^{\tau}+P_{\mathrm{FA}} z^{\tau+\tau_{c}}$, where $P_{\mathrm{FA}}$ is the FA probability, $z$ represents the unit delay operator, and $\tau_{c}$ denotes the penalty time [2]. Similarly, the transfer functions from the $i$ th in-phase cell to the acquisition state, and to the next cell, are given, respectively, by $H_{i}(z)=P_{\mathrm{D}_{i}} z^{\tau}$ and $M_{i}(z)=\left(1-P_{\mathrm{D} i}\right) z^{\tau}$, where $P_{\mathrm{D} i}$ represents the probability of detection for the $i$ th in-phase cell, for $i=1,2, \ldots, L$ (Fig. 2).

The MAT for a CSS algorithm can be calculated from MAT $=\left.\frac{1}{N} \sum_{j=1}^{N}\left[\frac{d \tilde{T}_{j}(z)}{d z}\right]\right|_{z=1}$, where $\tilde{T}_{j}(z)$ denotes the transfer function from cell $j$ to the acquisition state [2]. For a given set of in-phase cell indices, $\mathcal{L}=\left\{l_{1}, l_{2}, \ldots, l_{L}\right\}$, with $l_{1}=1$ without loss of generality, it can be shown, after some manipulation, that the MAT can be calculated as

$$
\text { MAT }=\left.\frac{1}{N} \sum_{i=1}^{L+1} \sum_{j=l_{i-1}+1}^{l_{i}}\left[\frac{d T_{i, j}(z)}{d z}\right]\right|_{z=1},
$$

where $l_{0} \doteq 0$ and $l_{L+1} \doteq N$ are defined for simplicity of the expressions, and

$$
T_{i, j}(z)=\left[H_{0}(z)\right]^{l_{i}-j}\left[G_{i}(z)+\frac{N_{i}(z) G_{1}(z)}{1-N_{1}(z)}\right],
$$

with

$$
\begin{aligned}
& G_{i}(z)=\sum_{m=i}^{L} H_{m}(z)\left[H_{0}(z)\right]^{l_{m}-l_{i}-m+i} \prod_{n=i}^{m-1} M_{n}(z), \\
& N_{i}(z)=\left[H_{0}(z)\right]^{N-l_{i}-L+i} \prod_{n=i}^{L} M_{n}(z) .
\end{aligned}
$$

Then, a generic expression for the MAT can be obtained from (8)-(10) as

$$
\begin{aligned}
& \text { MAT }=\frac{\left(N-l_{L}+1\right) \Gamma}{N\left(1-A_{1, L}\right)} \\
& +\frac{1}{N} \sum_{x=2}^{L}\left(l_{x}-l_{x-1}\right)\left[\left(l_{x}-l_{x-1}-1\right) \beta(x)+\theta(x)\right],
\end{aligned}
$$

where $A_{i, j} \doteq \prod_{n=i}^{j}\left(1-P_{\mathrm{D}_{n}}\right)$ for $i \leq j$ and $A_{i, j} \doteq 1$ for $i>j$,

$$
\begin{aligned}
\Gamma & \doteq \tau \sum_{i=0}^{L-1} A_{1, i}+\tau_{p} \sum_{i=1}^{L}\left(l_{i}-i+\frac{N-l_{L}}{2}\right) P_{\mathrm{D} i} A_{1, i-1} \\
& +\tau_{p}(N-L) A_{1, L} \\
\beta(x) & \doteq \frac{\tau_{p}}{2}\left(A_{x, L}+\sum_{i=x}^{L} P_{\mathrm{D}_{i}} A_{x, i-1}\right) \\
\theta(x) & \doteq \sum_{i=x}^{L} f_{i, x}(0,0) P_{\mathrm{D} i} A_{x, i-1}+\frac{A_{x, L}}{1-A_{1, L}} \\
\times & {\left[A_{1, L}\left[(N-L) \tau_{p}+L \tau\right]+\sum_{i=1}^{L} f_{i, x}(L, N-L) P_{\mathrm{D} i} A_{1, i-1}\right] }
\end{aligned}
$$

with $f_{i, x}(a, b) \doteq(i-x+1+a) \tau+\left(l_{i}-l_{x}-i+x+b\right) \tau_{p}$, and $\tau_{p} \doteq \tau+P_{\mathrm{FA}} \tau_{c}$.

Note that (12)-(15) provide closed-form expressions for the MAT calculation of any channel profile specified by $\mathcal{L}=\left\{l_{1}, l_{2}, \ldots, l_{L}\right\}$. 


\section{Special Cases}

In the case of a dense multipath channel specified by $\mathcal{L}=$ $\{1,2, \ldots, L\}$. the MAT in (12)-(15) can be simplified as

$$
\text { MAT }=\frac{(N-L+1) \Gamma}{N\left(1-A_{1, L}\right)}+\frac{1}{N} \sum_{x=2}^{L}\left[\frac{A_{x, L} \theta_{1}(x)}{1-A_{1, L}}+\theta_{2}(x)\right],
$$

where

$$
\begin{aligned}
& \Gamma=\tau \sum_{i=0}^{L-1} A_{1, i}+\frac{\tau_{p}}{2}(N-L)\left(1+A_{1, L}\right), \\
& \theta_{1}(x) \doteq \tau_{p}(N-L)+\tau(L-x+1)\left(1-A_{1, L}\right)+\tau \sum_{i=0}^{L-1} A_{1, i}, \\
& \theta_{2}(x) \doteq \tau \sum_{i=x}^{L}(i-x+1) P_{\mathrm{D} i} A_{x, i-1} .
\end{aligned}
$$

It is observed that this MAT formula is not exactly equal to that in [6] for dense multipath channels, since the derivation in that study assumes that the CSS can start only from the out-ofphase cells. Hence, the formulation in (16)-(17) removes that constraint to provide a more generic expression. Also note that the MAT formula in (16)-(17) reduces to that in [2] for a single path channel, i.e., for $L=1$.

Secondly, consider a scenario with $P_{\mathrm{D} i}=1$ for $i=$ $1,2, \ldots, L$, i.e., the in-phase cells can always be detected correctly. In fact, this scenario can approximate high signalto-noise ratio (SNR) situations for in-phase cell outputs. Then, the following MAT formula can be obtained from (12)-(15) as

$$
\begin{aligned}
\text { MAT } & =\frac{\left(N-l_{L}+1\right)}{N}\left[\tau+\frac{\tau_{p}}{2}\left(N-l_{L}\right)\right] \\
& +\frac{1}{N} \sum_{x=2}^{L}\left(l_{x}-l_{x-1}\right)\left[\left(l_{x}-l_{x-1}-1\right) \frac{\tau_{p}}{2}+\tau\right],
\end{aligned}
$$

which reduces to $\tau+0.5 \tau_{p}(N-L)(N-L+1) / N$ for dense channels.

Finally, for $N \gg L$ and $l_{i}-l_{i-1} \ll N, i=1,2, \ldots, L$, the MAT can approximated by

$\mathrm{MAT} \approx \frac{\tau_{p}}{1-A_{1, L}}\left[N A_{1, L}+\sum_{i=1}^{L}\left(l_{i}-i+\frac{N-l_{L}}{2}\right) P_{\mathrm{D} i} A_{1, i-1}\right]$,

which reduces to the MAT expression in [6] for dense channels (c.f. eqn. (4) in [6]). Note that the MAT formula in (19) provides an approximate result when the total number of cells, $N$, is considerably larger than the number of in-phase cells and the maximum distance between the in-phase cells.

\section{Simulation Results}

In this section, simulation results are presented in order to verify the theoretical analysis in the previous section. For the simulation scenario, the cell outputs are modeled as

$$
y_{i}=\left\{\begin{array}{ll}
\alpha_{i}+n_{i}, & i \in \tilde{\mathcal{L}} \\
n_{i}, & \text { otherwise }
\end{array},\right.
$$

where $\boldsymbol{\alpha}=\left[\begin{array}{ll}\alpha_{1} & \alpha_{2} \cdots \alpha_{L}\end{array}\right]$ represent the signal components in the in-phase cell outputs, and $n_{i}$ is the noise in the $i$ th cell

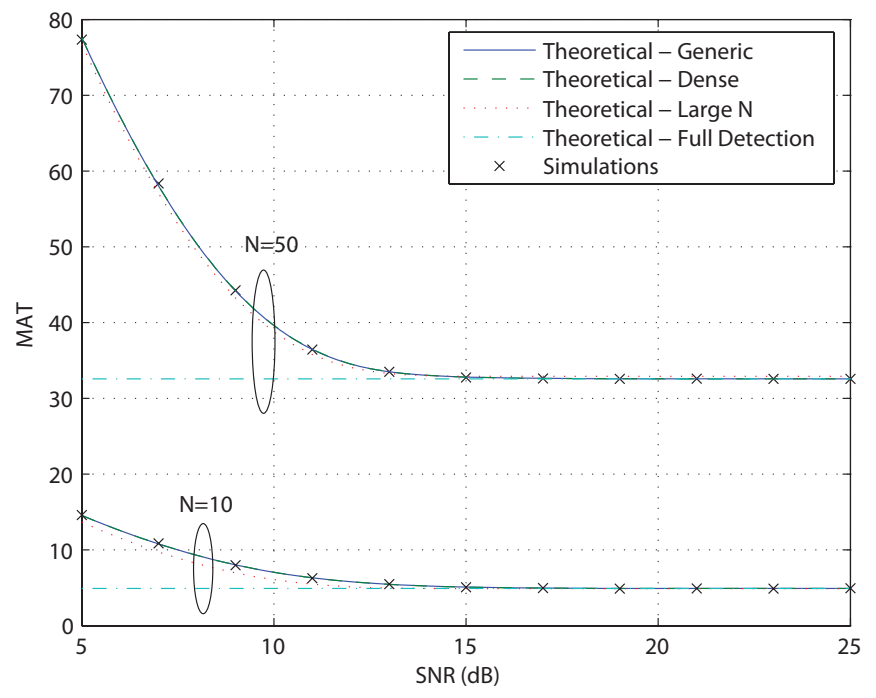

Fig. 3. Exact and approximate theoretical results and simulations results versus SNR for various numbers of cells in the uncertainty region.

output. In the simulations, $n_{i}$ 's are modeled as independent and identically distributed zero mean Gaussian random variables with variance $\sigma^{2}$. Then, in the acquisition process, the absolute value of $y_{i}$ is compared to a threshold $\delta$ in order to determine if the cell is an in-phase cell or not. In other words, the cell is declared as an in-phase cell if $\left|y_{i}\right|>\delta$, and as an out-of-phase cell otherwise. In order to have a systematic way of setting the threshold, the threshold is set to provide a desired FA probability $P_{\mathrm{FA}}$. Specifically, the threshold is determined from $\delta=\sigma Q^{-1}\left(0.5 P_{\mathrm{FA}}\right)$, where $Q^{-1}(x)$ is the inverse of the $Q$-function specified as $Q(x)=\frac{1}{\sqrt{2 \pi}} \int_{x}^{\infty} e^{-t^{2} / 2} \mathrm{~d} t$. In the simulations, $P_{\mathrm{FA}}=0.1$ is used to determine the threshold for a given noise level. For MAT calculations, it is assumed that testing of each cell takes one time unit (i.e., $\tau=1$ ), and each FA results in an additional 4 time units (i.e., $\tau_{c}=4$ ).

Note that the derivations in the previous section do not assume any probability distribution of the cell outputs or any specific cell testing technique. Therefore, the previous settings are selected only to provide an example scenario.

First, the exact and approximate MAT expressions in Section III are compared against the simulation results for a signal with 3 in-phase cells specified by $\boldsymbol{\alpha}=\left[\begin{array}{lll}1 & -0.9 & 0.75\end{array}\right]$ and $\tilde{\mathcal{L}}=\{1,2,3\}$. The curves in Fig. 3 illustrate the MATs for an FSSS-1 (i.e., CSS) algorithm for $N=10$ and $N=50$ cells in the uncertainty region. It is observed that the theoretical results obtained from (12)-(15) for the generic case and from (16)-(17) for dense channels match perfectly with the simulation results. On the other hand, the MAT expression in (18) gets accurate for high SNRs (labeled as 'Theoretical Full Detection' in Fig. 3), since the detection probabilities converge to 1 as the SNR increases, which is defined as $\mathrm{SNR}=10 \log \left(\frac{1}{\sigma^{2}} \sum_{i=1}^{L}\left|\alpha_{i}\right|^{2}\right)$. Finally, the asymptotic result in (19) for large number of cells in the uncertainty region, $N$, is observed to be quite accurate even for small $N$ values.

Next, the effects of the step size $M$ on the MAT are investigated for $N=101$ cells in the uncertainty region. A Nakagami-fading channel with 4 paths is considered, where 


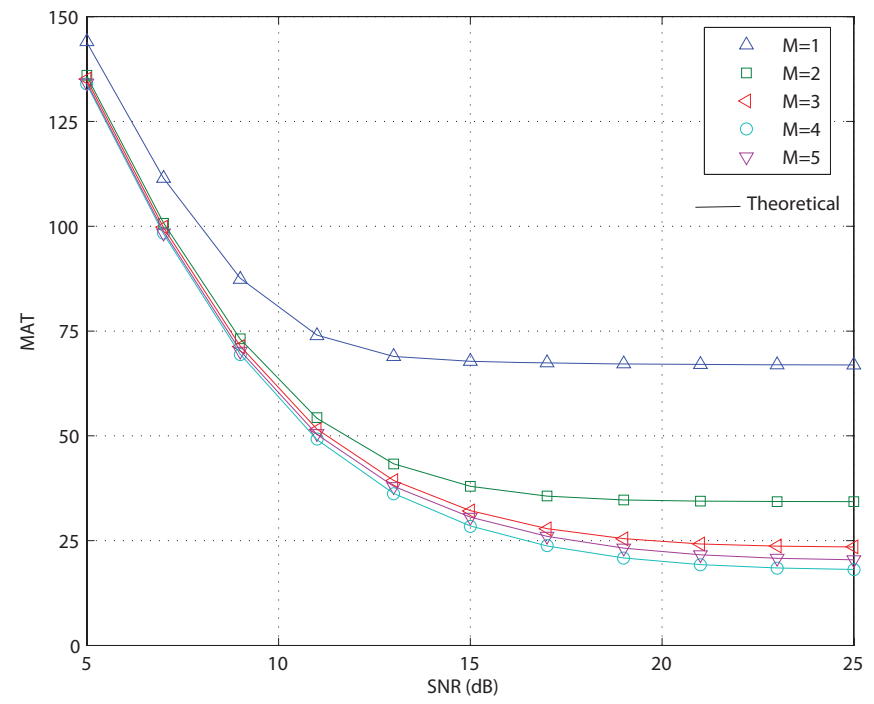

Fig. 4. MAT versus SNR for various step sizes and for a dense channel. Theoretical results and the simulations are in close agreement.

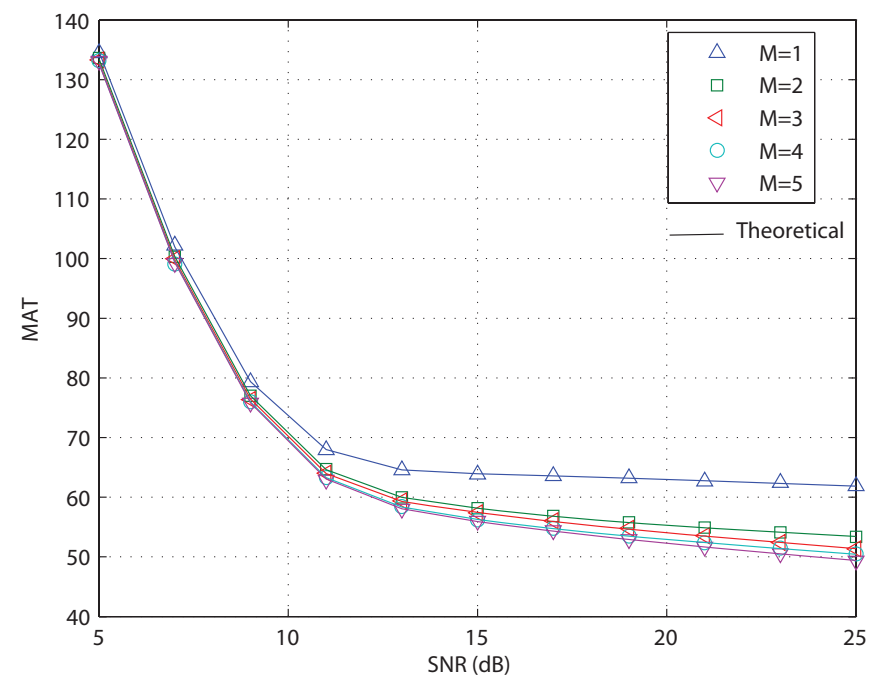

Fig. 5. MAT versus SNR for various step sizes and for a sparse channel. Theoretical results and the simulations are in close agreement.

the amplitude of path $i$ is distributed as a Nakagami- $m$ random variable with parameter $\Omega_{i}$. The average power of each path is modeled as exponentially decaying with its arrival time; i.e., $\Omega_{i} \propto \exp \{-i \lambda\}$ with $\lambda=0.5$ for $i=1,2,3,4$, and $m=3$ is employed. Fig. 4 illustrates the MATs obtained from the simulations and from (12)-(15) for various step sizes when $\tilde{\mathcal{L}}=\{1,2,3,4\}$ (averages over 100 channel realizations are considered). Note that the theoretical results are plotted via the solid lines and the geometric shapes representing the simulation results overlap perfectly with the theoretical lines. Note that $M=4$ provides the lowest MATs, which is in accordance with the common intuition that the optimal step size should be equal to the number of in-phase cells [8]. However, this is not true in all cases, as investigated below.

Fig. 5 considers the same parameter setting as in the previous case except that the delay differences between consecutive paths are modeled according to an exponential random

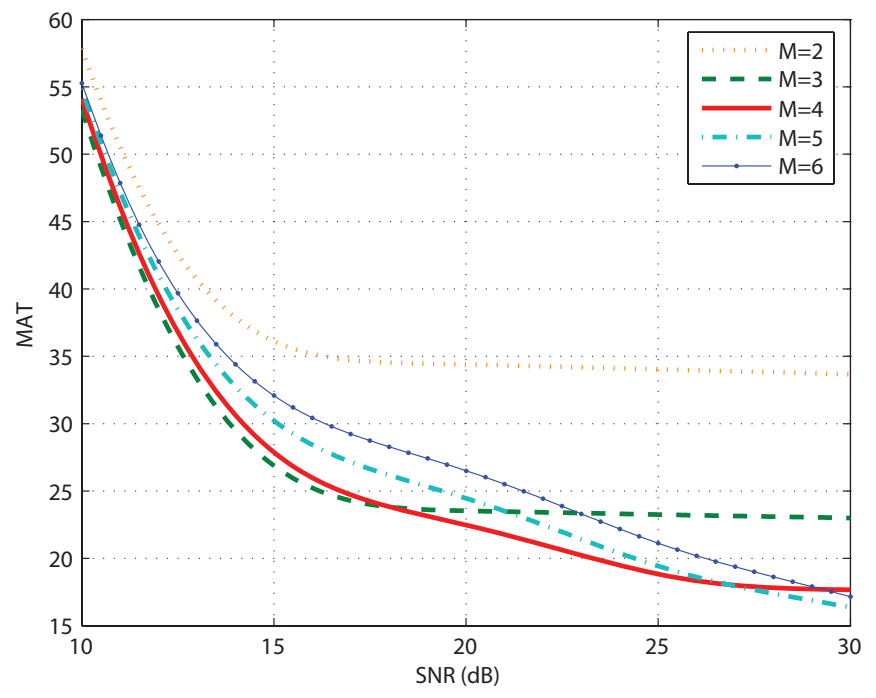

Fig. 6. MAT versus SNR for various step sizes and for a dense channel.

variable with a mean value of 10 cells (each delay value is rounded up to the next integer cell index). In other words, the channel is not assumed to be dense in this scenario. Similar to the previous case, a good agreement between theoretical and simulation results are observed. However, the optimal step size is not 4 anymore, which shows that the optimal step size is not necessarily given by the number of in-phase cells for sparse channels.

Finally, the MATs are plotted versus SNR for various step sizes when the system is specified by $N=101$,

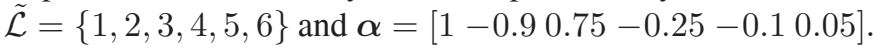
From Fig. 6, it is observed that the optimal step size varies with SNR. For example, at $\mathrm{SNR}=15 \mathrm{~dB}, M=3$ has a lower MAT than the others, whereas at $\mathrm{SNR}=20 \mathrm{~dB}, M=4$ is the one with the lowest MAT. Therefore, it is concluded that the optimal step size depends on both the channel profile and the SNR.

\section{COnClusions And Extensions}

Generic closed-form expressions for MATs of FSSS algorithms have been derived. First, it has been shown that the MAT of an FSSS algorithm is equal to that of a CSS algorithm when the in-phase cells are mapped according to the step size of the FSSS. Then, a generic MAT formula has been derived based on the flow graph approach. In addition, simple MAT expressions have been obtained for special cases. Finally, the simulations results have been presented to verify the theoretical analysis and to investigate the relation between system parameters and the MAT.

Note that the MAT formula in (6) is valid for any serial search algorithm when $\tilde{\mathbf{c}}_{i}$ 's are obtained according to that algorithm. In general, $\tilde{\mathbf{c}}_{i}$ 's are not circularly shifted versions of each other for algorithms other than the FSSS. Therefore, the MATs for such algorithms cannot simply be obtained from that of a CSS algorithm. However, it is straightforward to obtain $\mathrm{E}\left\{T \mid \tilde{\mathbf{c}}_{i}, \mathcal{L}_{\mathrm{r}}, \mathcal{U}\right\}$ similarly to the MAT derivations in Section III for each $\tilde{\mathbf{c}}_{i}$ and to obtain the MAT from the generic expression in (6), which, however, results in an implicit expression. 


\section{ACKNOWLEDGMENTS}

The author would like to thank Watcharapan Suwansantisuk for the useful discussions.

\section{REFERENCES}

[1] R. E. Ziemer and R. L. Peterson, Digital Communications and Spread Spectrum Systems. New York: Macmillan, 1985.

[2] A. Polydoros and C. L. Weber, "A unified approach to serial search spread-spectrum code acquisition-part I: general theory," IEEE Trans. Commun., vol. COM-32, pp. 542-549, May 1984.

[3] E. A. Homier and R. A. Scholtz, "Rapid acquisition of ultra-wideband signals in the dense multipath channel," in Proc. IEEE Conference on Ultra Wideband Systems and Technologies, pp. 105-109, May 2002.

[4] S. Vijayakumaran and T. F. Wong, "A search strategy for ultra-wideband signal acquisition," IEEE Trans. Commun., vol. 53, no. 12, pp. 2015 2019, Dec. 2005
[5] W. Suwansantisuk and M. Z. Win, "Multipath aided rapid acquisition: optimal search strategies," IEEE Trans. Inform. Theory, vol. 53, no. 1, pp. 174-193, Jan. 2007.

[6] L.-L. Yang and L. Hanzo, "Serial acquisition of DS-CDMA signals in multipath fading mobile channels," IEEE Trans. Veh. Technol., vol. 50, no. 2, pp. 617-628, March 2001.

[7] W. Suwansantisuk, M. Z. Win, and L. A. Shepp, "On the performance of wide-bandwidth signal acquisition in dense multipath channels," IEEE Trans. Veh. Technol., vol. 54, no. 5, pp. 15841594, Sept. 2005.

[8] E. A. Homier and R. A. Scholtz "Hybrid fixed-dwell-time search techniques for rapid acquisition of ultra-wideband signals," in Proc. International Workshop on Ultra Wideband System Oulu, Finland, June 2003.

[9] S. Vijayakumaran and T. F. Wong, "On equal gain combining for acquisition of time-hopping ultra-wideband signals," IEEE Trans. Commun., vol. 54, no. 3, pp. 479-490, Mar. 2006. 